\title{
Phase-change-driven dielectric-plasmonic transitions in chalcogenide metasurfaces
}

\author{
Behrad Gholipour ${ }^{1,2}$, Artemios Karvounis ${ }^{1}$, Jun Yin ${ }^{3}$, Cesare Soci ${ }^{3}$, Kevin F. MacDonald ${ }^{1}$ and Nikolay I. Zheludev ${ }^{1,3}$
}

\begin{abstract}
Chalcogenides-alloys based on group-16 'chalcogen' elements (sulfur, selenium, and tellurium) covalently bound to 'network formers' such as arsenic, germanium, antimony, and gallium - have a variety of technologically useful properties, including infrared transparency, high optical nonlinearity, photorefractivity and readily induced, reversible, non-volatile structural phase switching. Such phase-change materials are of enormous interest in the fields of plasmonics and nanophotonics. However, in such applications, the fact that some chalcogenides accrue plasmonic properties in the transition from an amorphous to a crystalline state, i.e., the real part of their relative permittivity becomes negative, has gone somewhat unnoticed. Indeed, one of the most commercially important chalcogenide compounds, germanium antimony telluride $\left(\mathrm{Ge}_{2}: \mathrm{Sb}_{2}: \mathrm{Te}_{5}\right.$ or GST), which is widely used in rewritable optical and electronic data storage technologies, presents this behavior at wavelengths in the near-ultraviolet to visible spectral range. In this work, we show that the phase transition-induced emergence of plasmonic properties in the crystalline state can markedly change the optical properties of sub-wavelength-thickness, nanostructured GST films, allowing for the realization of non-volatile, reconfigurable (e.g., color-tunable) chalcogenide metasurfaces operating at visible frequencies and creating opportunities for developments in non-volatile optical memory, solid state displays and alloptical switching devices.
\end{abstract}

\section{Introduction}

Phase-change materials have had a significant role in the evolution of active plasmonic and photonic metamaterial technologies, delivering a variety of switchable, tunable, and reconfigurable optical functionalities through hybridization with plasmonic metal nanostructures $^{1-5}$. Chalcogenides, which can be electrically and optically switched between amorphous and crystalline states with markedly different electronic and photonic properties $^{6-10}$, have facilitated the realization of active plasmonic metamaterial devices for a variety of applications including electro- and all-optical signal switching, polarization modulation, beam steering, and

Correspondence: Behrad Gholipour (b.gholipour@soton.ac.uk) or Kevin F. MacDonald (kfm@orc.soton.ac.uk)

'Optoelectronics Research Centre \& Centre for Photonic Metamaterials, University of Southampton, Highfield, Southampton, SO17 1BJ, UK

${ }^{2}$ School of Chemistry, University of Southampton, Highfield, Southampton, SO17 1BJ, UK

Full list of author information is available at the end of the article multispectral imaging ${ }^{11-19}$. Moreover, the near-infrared high refractive index and index contrast between phase states within germanium antimony telluride $\left(\mathrm{Ge}_{2}: \mathrm{Sb}_{2}: \mathrm{Te}_{5}\right.$ or GST) have recently been harnessed in the demonstration of laser-rewritable and optically switchable, nanostructured 'all-dielectric' (i.e., all-chalcogenide) metasurfaces ${ }^{20-22}$. At the same time, several bulk monocrystalline chalcogenides have been recognized as 'topological insulators' (TIs)-semiconductors with topologically protected metallic surface states arising through strong spin-orbit interactions ${ }^{23}$. Indeed, the quaternary $\mathrm{TI}$ alloy $\mathrm{Bi}_{1.5} \mathrm{Sb}_{0.5} \mathrm{Te}_{1.8} \mathrm{Se}_{1.2}$ (BSTS) has recently been demonstrated as a UV-visible range plasmonic medium ${ }^{25}$.

Here, we show that thin-film GST can be optically switched between amorphous and polycrystalline states, which are dielectric and metallic (i.e., plasmonic) respectively at UV/VIS wavelengths, and demonstrate this switching in the context of photonic metasurfaces.

\section{(c) The Author(s) 2018



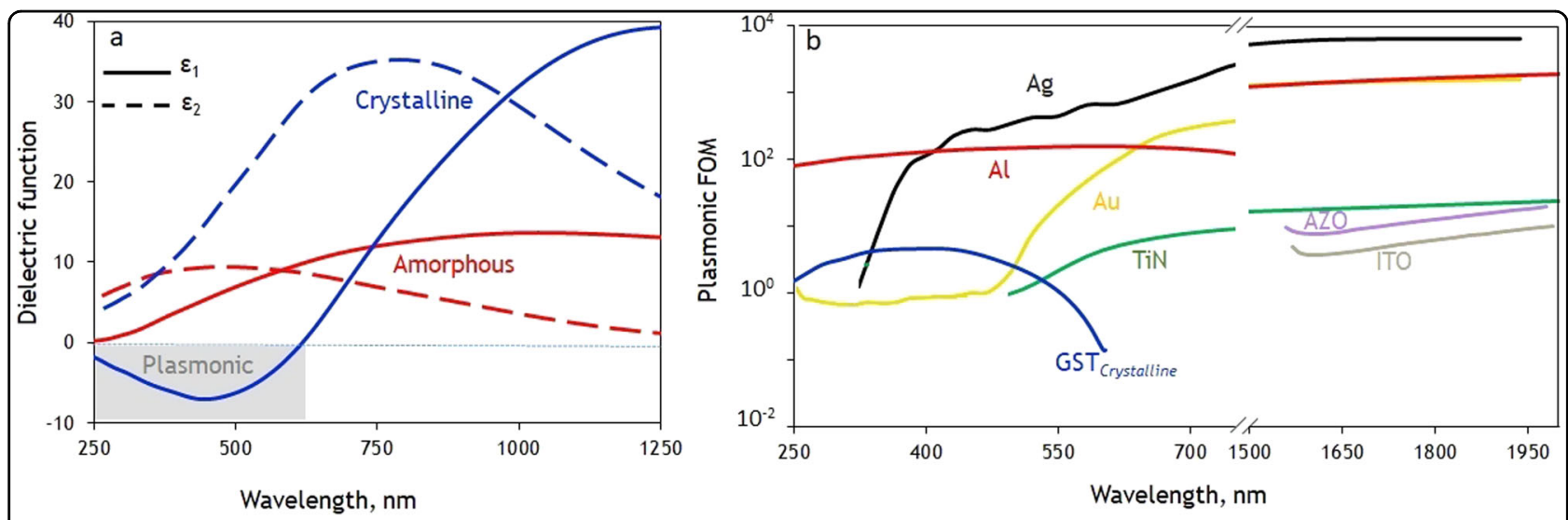

Fig. 1 Chalcogenide plasmonics. a Spectral dispersion [from variable angle ellipsometric measurements] of the real $\varepsilon_{1}$ and imaginary $\varepsilon_{2}$ parts of the relative permittivity of sputtered germanium antimony telluride [GST] in its amorphous and polycrystalline phases. $\mathbf{b}$ Spectral dispersion of plasmonic figures of merit for polycrystalline GST and a selection of other plasmonic materials [as labeled; calculated using material parameters from refs. ${ }^{24,40}$ ]

The real part $\varepsilon_{1}$ of the relative permittivity (Fig. 1a) of polycrystalline GST has a negative value at wavelengths $\lambda$ below $660 \mathrm{~nm}$, as is required for a medium in air/ vacuum to support surface plasmons ${ }^{26}$, and a positive value at longer wavelengths. In contrast, amorphous GST is dielectric with a positive value of $\varepsilon_{1}$ across the entire ultraviolet to near-infrared spectral range. Figure $1 \mathrm{~b}$ presents the plasmonic figures of merit $\operatorname{Re}\left\{k_{S P P}\right\} / 2 \pi \operatorname{Im}\left\{k_{S P P}\right\}$-the surface plasmon polariton (SPP) propagation decay length in units of SPP wavelength ${ }^{27}$-for polycrystalline GST alongside a number of other, noble metals and recently proposed alternative ${ }^{28}$ plasmonic media. $\left(k_{S P P}=k_{0} \sqrt{\varepsilon_{m} \varepsilon_{d} /\left(\varepsilon_{m}+\varepsilon_{d}\right)}\right.$ is the wavevector of the surface plasmon polaritons on a planar interface between the metallic and dielectric media with complex relative permittivities of $\varepsilon_{\mathrm{m}}$ and $\varepsilon_{\mathrm{d}}$, respectively; the latter is taken to be air in the present case; $k_{0}$ is the free space wavevector). On this basis, GST is surpassed only by $\mathrm{Al}$ in the near-UV spectral range and is comparable as a plasmonic medium for UV and blue/green visible wavelengths to highly doped transparent conductive oxides at near-infrared wavelengths.

In isolation, optically thick GST is an absorbing medium at ultraviolet/visible wavelengths with a dispersion of reflectivity that can be substantially modified by nano-grating metasurface structures, yielding vibrant plasmonic colors in the crystalline state when illuminated with light polarized perpendicular to the grating lines (see Supplementary Figure S1). For the present study however, we adopt an encapsulated layer structure closer to that which would ultimately be required in a practical reversible switching device, whereby a thin chalcogenide film is protected against atmospheric degradation, especially at elevated phase-transition temperatures ${ }^{11}$. While this encapsulation does have the effect of suppressing absolute levels of reflectivity and transmission relative to those of a free-standing thin film (a result of Fabry-Perot-type interference between the central high-index absorbing film and lower-index layers either side ${ }^{29}$ ), it nonetheless facilitates a demonstration of an optically induced change in the character of the chalcogenide, from dielectric to plasmonic.

\section{Materials and methods}

For the present study, $70 \mathrm{~nm}$ of GST was deposited between $70 \mathrm{~nm}$ protective layers of $\mathrm{ZnS} / \mathrm{SiO}_{2}$ on an optically flat quartz substrate by RF sputtering (Kurt J. Lesker Nano 38) from $\mathrm{Ge}_{2} \mathrm{Sb}_{2} \mathrm{Te}_{5}$ and $\mathrm{ZnS}: \mathrm{SiO}_{2}$ (1:9) alloy targets. A base pressure of $5 \times 10^{-7}$ mbar was achieved prior to the deposition, and high-purity argon was used as the sputtering gas. The substrate was held within $10 \mathrm{~K}$ of room temperature on a rotating plate 150 $\mathrm{mm}$ from the target to produce low-stress amorphous films. The chemical composition of the deposited phasechange film was confirmed using energy dispersive Xray spectroscopy (Supplementary Figure S2). Nanograting metasurface arrays were subsequently milled though the $\mathrm{ZnS} / \mathrm{SiO}_{2}-\mathrm{GST}-\mathrm{ZnS} / \mathrm{SiO}_{2}$ tri-layer using a focused (gallium)-ion beam (FEI Helios NanoLab 600) at beam currents $\leq 28 \mathrm{pA}$ to prevent crystallization of the GST (via ion beam-induced heating) during the milling process. Metasurface domains, each measuring $20 \times 20$ $\mu \mathrm{m}$ in the sample plane, were fabricated with a range of periods, $P$, from 250 to $400 \mathrm{~nm}$ at a fixed milled linewidth, $W$, of $100 \mathrm{~nm}$ (Fig. 2).

The amorphous-to-crystalline transition in chalcogenides is an annealing process that may be initiated across the whole sample by increasing the ambient temperature or locally by laser- or electrical currentinduced heating to a temperature above the material's 


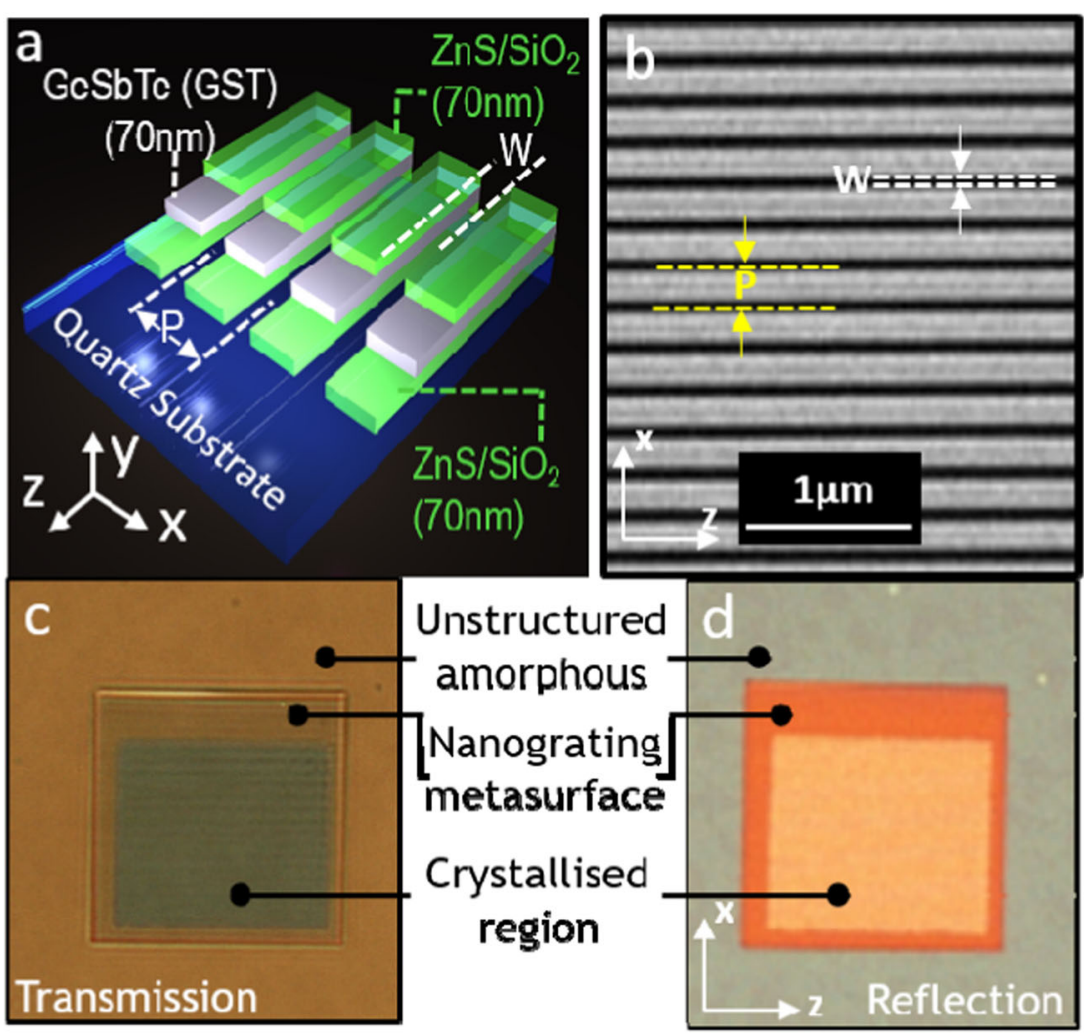

Fig. 2 GST metasurfaces. a Artistic cut-away section of the nanograting metasurface structures fabricated with a $70 \mathrm{~nm}$ film of GST between two layers [70 nm each] of $\mathrm{ZnS} / \mathrm{SiO}_{2}$. b Scanning electron microscopy image of a section of a $\mathrm{ZnS} / \mathrm{SiO}_{2}-\mathrm{GST}-\mathrm{ZnS} / \mathrm{SiO}_{2}$ tri-layer metasurface [dark areas are the quartz substrate exposed by focused ion-beam milling, i.e., removal of the tri-layer]. c Transmission and $\mathbf{d}$ reflection TM-polarized, normalincidence reflection images of a $20 \times 20 \mu \mathrm{m}, P=400 \mathrm{~nm}$, amorphous phase, GST, tri-layer metasurface domain [surrounded by unstructured, amorphous phase GST tri-layer], part of which—the central $12 \times 12 \mu \mathrm{m}$ region appearing green in transmission mode and pale orange in reflection mode-has been selectively crystallized by fs-pulsed laser illumination

glass-transition point, $T_{\mathrm{g}}\left(\sim 160^{\circ} \mathrm{C}\right.$ for GST), but below its melting point, $T_{\mathrm{m}}\left(\sim 600^{\circ} \mathrm{C}\right)^{30}$. The reverse transition -a melt-quenching process-can be driven by shorter, higher energy pulsed excitation that momentarily brings the material to a temperature above $T_{\mathrm{m}}{ }^{31}$. In this work, structural transitions in the GST layer were excited using $85 \mathrm{fs}$ laser pulses at a wavelength of $730 \mathrm{~nm}$ with a beam focused to a diffraction-limited spot and rasterscanned over the sample using a spatial light modulator, as described in ref. ${ }^{32}$ By varying the number, repetition rate and energy of the pulses delivered at a given point, one may accurately control the temporal profile of the optically induced temperature change in the GST. Here, we employed trains of fifty $\sim 140 \mathrm{~mJ} \mathrm{~cm}^{-2}$ pulses at a repetition rate of $1 \mathrm{MHz}$ for crystallization.

The normal-incidence transmission and reflection characteristics of the GST nanograting metasurfaces in amorphous and crystalline states were quantified for incident polarizations perpendicular and parallel to the grating lines (along the $x$ and $z$ directions defined in Fig. 2 or the TE and TM orientations of the grating, respectively) using a microspectrophotometer (CRAIC QDI2010) with a sampling domain size of $15 \mu \mathrm{m} \times 15 \mu \mathrm{m}$ and numerical aperture of 0.28 .

\section{Results and discussion}

For GST in the amorphous phase, the transmission of the unstructured $\mathrm{ZnS} / \mathrm{SiO}_{2}-\mathrm{GST}-\mathrm{ZnS} / \mathrm{SiO}_{2}$ tri-layer increases monotonically with the wavelength across the UV-to-near-IR spectral range, from $\sim 5 \%$ at $400 \mathrm{~nm}$ to $35 \%$ at $900 \mathrm{~nm}$ (Fig. 3a). For the polycrystalline phase, transmission levels are suppressed across the entire range but follow essentially the same trend, reaching $\sim 12 \%$ at $900 \mathrm{~nm}$ (Fig. 3b). The sub-wavelength period (i.e., non-diffractive) nanograting metasurface structures introduce resonances for TM-polarized light (incident electric field perpendicular to the grating lines) at visible wavelengths depending on the period, $P$, for both phase states of GST, which manifest themselves in the transmitted and reflected colors of the metamaterial domains (Fig. 2c, d). Owing to the highly anisotropic nature of nanograting resonators the optical response 

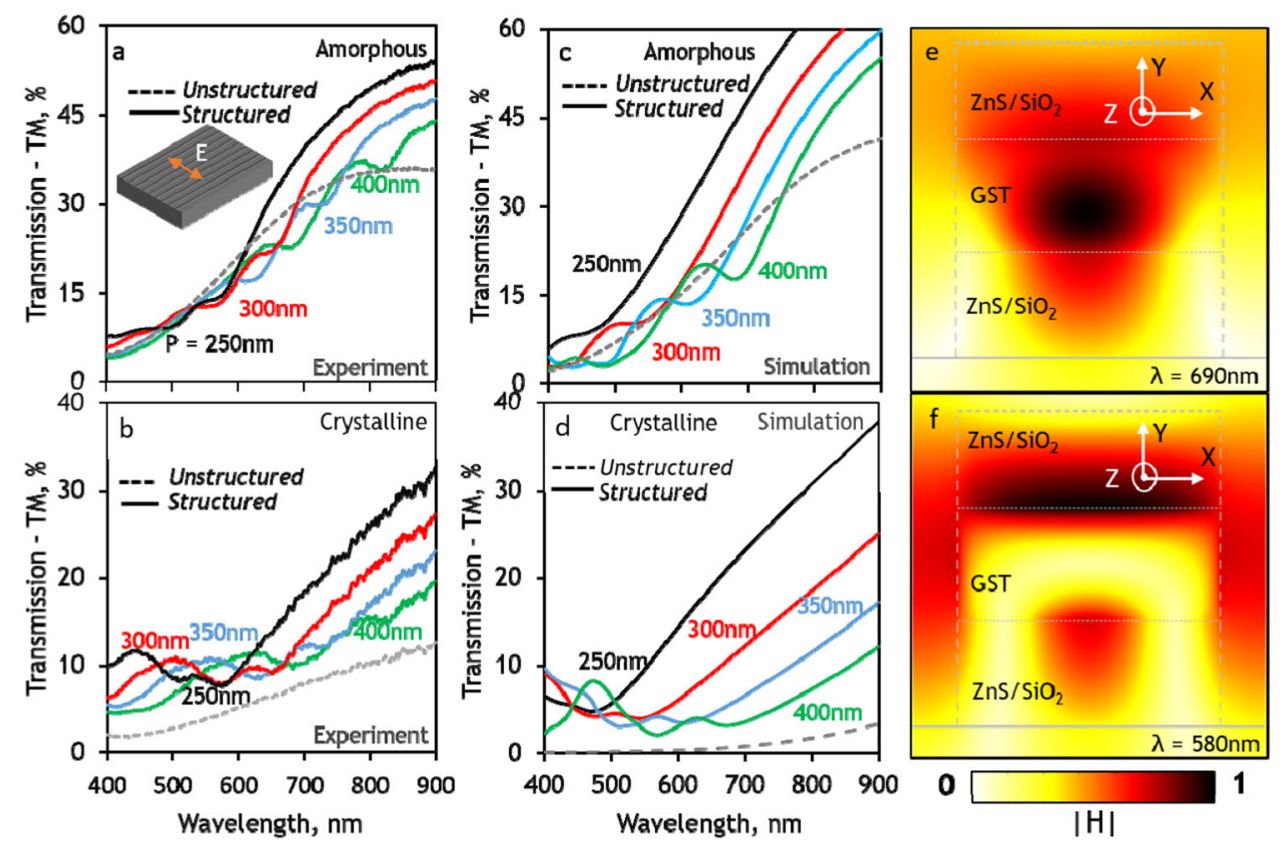

Fig. 3 Optical properties of amorphous [dielectric] and polycrystalline [plasmonic] GST metasurfaces. a, b Measured spectral dispersion of $\mathrm{ZnS} / \mathrm{SiO}_{2}-\mathrm{GST}-\mathrm{ZnS} / \mathrm{SiO}_{2}$ nanograting metasurface TM transmission for a selection of grating periods, $P$ [as labeled], for the amorphous (a) and polycrystalline (b) states of the GST layer overlaid with spectra for the unstructured tri-layer [dashed lines]. Corresponding numerically simulated transmission spectra (c, d). Distributions of the normalized magnetic field, $H$, in the $x z$-plane for a unit cell of a $P=400 \mathrm{~nm}$ nanograting $(\mathbf{e}, \mathbf{f})$ for a transmission resonance wavelength at $\lambda=580 \mathrm{~nm}$ for amorphous and $690 \mathrm{~nm}$ for the crystalline phase

depends on the polarization of the incident light and the resonant peaks disappear with incident polarization parallel to the grooves of the grating (TE). In this orientation, the nanograting behaves as a medium with a non-dispersive effective refractive index related to that of the $\mathrm{ZnS} / \mathrm{SiO}_{2}-\mathrm{GST}-\mathrm{ZnS} / \mathrm{SiO}_{2}$ stack and its fill fraction within the grating structure. As such, the observed optical response in either amorphous or polycrystalline GST is largely invariant with the nanograting period and almost identical to that of the unstructured tri-layer transmission spectrum for the corresponding phase state. (Corresponding reflection spectra and TEpolarized spectra are presented in Supplementary Figure S3 and S4, respectively).

It should be noted that the observed TM resonances are markedly different for the two phase states of GST. For the amorphous phase, they are displacement current resonances reliant upon the high refractive index contrast between the dielectric GST and the surrounding media, whereas for the crystalline phase, the resonances are plasmonic, i.e., based upon the opposing signs of $\varepsilon_{1}$ at the interfaces between the GST and surrounding media. Numerical simulations clearly illustrate this difference. Figure $3 \mathrm{c}-\mathrm{f}$ shows the results of a 3D finite-element Maxwell solver model, which employs ellipsometrically measured values for the complex permittivity of GST, as presented in Fig. 1a. This model assumes lossless non-dispersive refractive indices of 1.46 and 1.80 for the semi-infinite quartz substrate and the $\mathrm{ZnS} / \mathrm{SiO}_{2}$ layers, respectively, normal incident narrowband plane-wave illumination, and due to the periodic boundary conditions, a nanograting pattern of infinite extent in the $x-z$ plane. There is good qualitative and quantitative agreement between the experimentally measured (Fig. 3a, b) and numerically simulated (Fig. 3c, d) transmission spectra for the GST metasurfaces in both amorphous and crystalline states. Discrepancies can be attributed to manufacturing imperfections (the computational model assumes an ideal, rectilinear nanograting geometry, as illustrated schematically in Fig. 2a) and contamination of the $\mathrm{ZnS} / \mathrm{SiO}_{2}$ and GST layers (gallium-ion implantation) during FIB milling, which slightly modifies the refractive index. The cross-sectional distributions of the magnetic field in Fig. 3e and $f$ reveal the essential differences between the resonant modes for the amorphous and crystalline phase of GST. In the former case, the field is stronger within the GST layer (higher index dielectric) than the $\mathrm{ZnS} / \mathrm{SiO}_{2}$ layers above and below, while in the latter, the field is 'expelled' from the body of the metallic GST layer and is stronger at the interfaces with $\mathrm{ZnS} / \mathrm{SiO}_{2}$. 

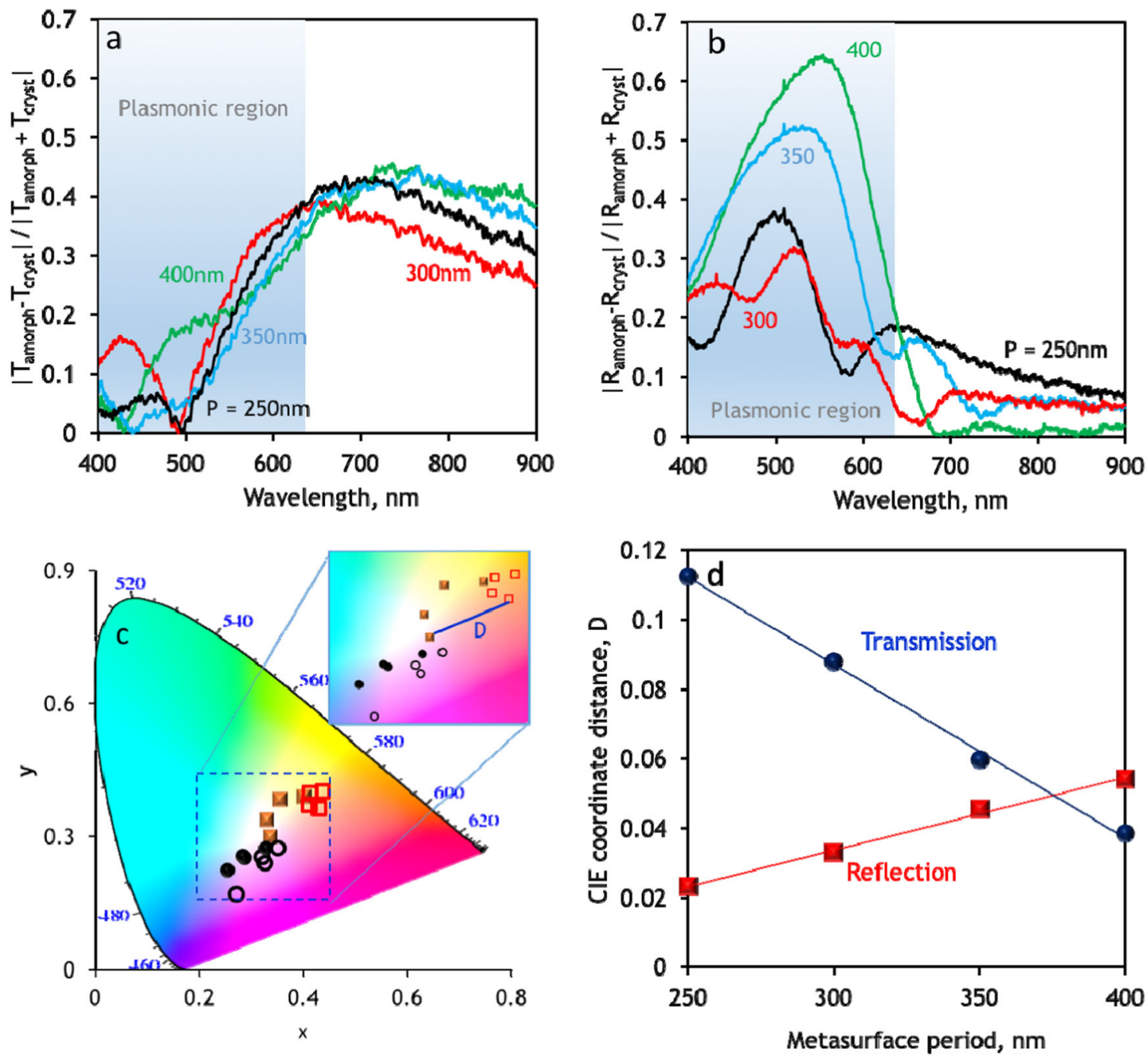

Fig. 4 Swiching contrast and color change. Relative $\mathbf{a}$ transmission and $\mathbf{b}$ reflection changes induced by the structural change between the amorphous and crystalline phases of the GST metasurfaces with different periods (as labeled). c Corresponding reflection (circles) and transmission (squares) CIE color palettes with the marked points indicating the metasurfaces $[P=250-400 \mathrm{~nm}]$ shown for both the amorphous (unfilled) and crystalline (filled) phases. $\mathbf{d}$ Color change resulting from the phase transition quantified as the Euclidean distance $[D]$ in the CIE color plot between corresponding amorphous and crystalline points, given as a function of the metamaterial period

Non-volatile, light-induced structural transitions between the amorphous and crystalline phases of GST, i.e., conversion between dielectric and plasmonic states, change the transmission and reflection characteristics of the metamaterial, which are manifested in the visible spectral range as changes in color. We define the transmission and reflection switching contrast as a ratio of the difference between the levels of the amorphous and crystalline phases of the GST layer to the sum of the levels (Fig. 4a, b). In the present case, the greatest contrast is achieved in reflection at grating period-dependent wavelengths in the spectral band where GST is converted between dielectric and plasmonic forms. The associated changes in color are not though a straightforward function of (resonant) contrast, they relate to changes in the dispersion of reflectivity and transmission integrated over the entire visible range, and can be quantified within the CIE 1931 color space. The color coordinates for the GST tri-layer metasurfaces in their amorphous and crystalline phases were derived directly from the reflection and transmission spectra (using Judd-Vosmodified CIE 2-deg color matching functions ${ }^{33}$ assuming in all cases an illuminating light source with a spectral radiant power distribution of a $6500 \mathrm{~K}$ black body and normalized observational brightness level) and are plotted in a two-dimensional representation of the CIE 1931 space in Fig. 4c. The change in color brought about by the structural transition in GST can be quantified as the Euclidean distance between the corresponding points in 
the CIE space (Fig. 4d) and is remarkably found to be a linear function of the metasurface period, $P$, for both transmission and reflection. The magnitude of the color change, in these terms, increases with period for reflection and decreases with period for transmission. From a physical point of view, this may be understood to result from a combination of the fact that as the period of the metasurface increases, both reflection and transmission resonances red-shift with a diminishing resonance quality-factor $(\mathrm{Q})$ and that the amorphous to crystalline phase transition broadly increases reflection and decreases transmission. So in reflection, while resonance $\mathrm{Q}$ decreases with increasing period, against the background of increasing reflectivity upon crystallization, the magnitude of CIE coordinate change with structural phase increases. Conversely, in transmission, decreasing $\mathrm{Q}$ with increasing period, in tandem with a decrease in transmission upon crystallization, results

in a decrease in the magnitude of change in CIE coordinate.

In summary, we have shown that switching of the chalcogenide phase-change medium GST between its amorphous and crystalline phases delivers a non-volatile change between dielectric (positive real part of the relative permittivity $\varepsilon$ ) and metallic (negative real part of $\varepsilon$ ) character in the UV-visible spectral range. This behavior enables switching between all-dielectric and plasmonic resonances in nanostructured GST in a sub-wavelength, optically switchable device geometry providing non-volatile changes in transmitted and reflected color. Phase-change chalcogenides have been proposed as potential candidates for future solid-state displays. To obtain the color gamut required, research in this direction has often focused on multilayer (nano)structures in which colour is a designed function of chalcogenide and/or spacer layer thicknesses ${ }^{34-37}$. On the other hand, the color variations presented by nanograting metasurfaces are achieved with a fixed layer thickness via in-plane structural design at the sub-wavelength scale. Patterns may be engineered to be dependent or independent of the polarization of incident light based (e.g., in the latter case through use of square pillars or circular nanodisk designs). This nanostructural approach can also be used in conjunction with thin-film interference effects, and when optimized through selection of appropriate layer thicknesses can deliver absorptive, transmissive or reflective devices ${ }^{38}$.

For the purpose of this proof-of-principle demonstration, we chose a commonly used GST composition $\left(\mathrm{Ge}_{2}: \mathrm{Sb}_{2}\right.$ : $\mathrm{Te}_{5}$ ); however, it should be noted that among potential material platforms for photonic metamaterials GST, and more broadly the chalcogenides, offer unique compositional variety (i.e., range and variability of material parameters) upon changes in stoichiometry, offering the possibility of improved losses and changes in the index with a change in the composition ${ }^{39}$. Furthermore, they are capable of providing high-index dielectric, 'epsilon-near-zero' (ENZ) or topological insulator properties when the constituent elements are combined in the right proportion.

The parameter space for the metasurface design is large, and different structural designs can extend the accessible color range beyond those demonstrated here, giving several extra degrees of freedom in the design of phase-change color pixels. Furthermore, such metasurfaces can be produced over large areas using techniques such as nanoimprint lithography, micro-contact printing or nanoembossing. These scalable manufacturing alternatives to ion-beam milling and reactive etching techniques will also minimize process-related degradation and contamination in the active phase-change layer.

Consequently, such color-tunable metasurfaces offer a robust CMOS (complementary metal-oxide-semiconductor) process compatible material platform for active and reconfigurable metadevices that may find application in thin solid-state displays, data storage, switchable/tunable filters, beam shapers, and optical limiting components.

\section{Data availability}

Following a period of embargo, the data from this paper can be obtained from the University of Southampton ePrints research repository: https://doi.org/10.5258/ SOTON/D0449.

\section{Acknowledgements \\ The work is supported by the UK Engineering and Physical Sciences Research Council [Grants EP/M009122/1 and EP/N00762X/1] and the Ministry of Education, Singapore [Grants MOE2011-T3-1-005 and MOE2016-T3-1-006]. \\ Author details \\ ${ }^{1}$ Optoelectronics Research Centre \& Centre for Photonic Metamaterials, University of Southampton, Highfield, Southampton, SO17 1BJ, UK. ${ }^{2}$ School of Chemistry, University of Southampton, Highfield, Southampton, SO17 1BJ, UK. ${ }^{3}$ Centre for Disruptive Photonic Technologies, TPI \& SPMS, Nanyang Technological University, 21 Nanyang Link, Singapore, 637371, Singapore}

\section{Authors' contributions}

B.G. generated the idea and designed, and carried out the experiments. A.K. performed the modeling. C.S. and J.Y. performed the DFT modeling. All authors contributed to the interpretation of the results. B.G., N.I.Z., and K.F.M. wrote the manuscript. N.I.Z. supervised the work.

Conflict of interest

The authors declare that they have no conflict of interest.

\section{Publisher's note}

Springer Nature remains neutral with regard to jurisdictional claims in published maps and institutional affiliations.

Supplementary information is available for this paper at https://doi.org/ 10.1038/s41427-018-0043-4.

Received: 20 October 2017 Revised: 9 February 2018 Accepted: 5 March 2018.

Published online: 11 June 2018 


\section{References}

1. MacDonald, K. F., Krasavin, A. V. \& Zheludev, N. I. Optical modulation of surface plasmon-polariton coupling in a gallium/aluminium composite. Opt. Commun. 278, 207-210 (2007).

2. Driscoll et al. Memory metamaterials. Science 325, 1176580 (2009).

3. Dicken, M. J. et al. Frequency tunable near-infrared metamaterials based on VO2 phase transition. Opt. Express 17, 18330 (2009).

4. Sámson, Z. L. et al. Metamaterial electro-optic switch of nanoscale thickness. Appl. Phys. Lett. 96, 143105 (2010).

5. Ahmadivand, A., Gerislioglu, B., Sinha, R., Karabiyik, M. \& Pala, N. Optical switching using transition from dipolar to charge transfer plasmon modes in Ge2Sb2Te5 bridged metallodielectric dimers. Sci. Rep. 7, 42807 (2017).

6. Shportko, K. et al. Resonant bonding in crystalline phase-change materials. Nat. Mater. 7, 653-658 (2008).

7. Lee, T. H. \& Elliott, S. R. The relation between chemical bonding and ultrafast crystal growth. Adv. Mater. 29, 1700814 (2017).

8. Borisenko, K. B. et al. Photo-induced optical activity in phase-change memory materials. Sci. Rep. 5, 8770 (2015).

9. Gholipour, B., Huang, C.-C., Ou, J.-Y. \& Hewak, D. W. Germanium antimony lateral nanowire phase change memory by chemical vapor deposition. Phys. Status Solidi 250, 994-998 (2013).

10. Hewak, D. \& Gholipour, B. Primed to remember. Science 336, 1515-1516 (2012).

11. Gholipour, B., Zhang, J., MacDonald, K. F., Hewak, D. W. \& Zheludev, N. I. An alloptical, non-volatile, bidirectional, phase-change meta-switch. Adv. Mater. 25 3050-3054 (2013).

12. Michel, A.-K. U. et al. Using low-loss phase-change materials for mid-infrared antenna resonance tuning. Nano Lett. 13, 3470-3475 (2013).

13. Chen, Y. G. et al. Hybrid phase-change plasmonic crystals for active tuning of lattice resonances. Opt. Express 21, 13691 (2013).

14. Tittl, A. et al. A switchable mid-infrared plasmonic perfect absorber with multispectral thermal imaging capability. Adv. Mater. 27, 4597-4603 (2015).

15. Rude, $\mathrm{M}$. et al. Optical switching at $1.55 \mathrm{um}$ in silicon racetrack resonators using phase change materials. Appl. Phys. Lett. 103, 141119 (2013).

16. Cao, T., Zheng, G., Wang, S. \& Wei, C. Ultrafast beam steering using gradient Au- Ge_2Sb_2Te_5 -Au plasmonic resonators. Opt. Express 23, 18029 (2015).

17. Cao, T., Zhang, L., Simpson, R. E. \& Cryan, M. J. Mid-infrared tunable polarization-independent perfect absorber using a phase-change metamaterial. J. Opt. Soc. Am. B 30, 1580 (2013).

18. Wuttig, M., Bhaskaran, H. \& Taubner, T. Phase-change materials for non-volatile photonic applications. Nat. Photonics 11, 465-476 (2017).

19. Raeis-Hosseini, N. \& Rho, J. Metasurfaces based on phase-change material as a reconfigurable platform for multifunctional devices. Materials 10, 1046 (2017).

20. Wang, Q. et al. Optically reconfigurable metasurfaces and photonic devices based on phase change materials. Nat. Photonics 10, 60-65 (2015).

21. Karvounis, A., Gholipour, B., MacDonald, K. F. \& Zheludev, N. I. directionAlldielectric phase-change reconfigurable metasurface. Appl. Phys. Lett. 109, 051103 (2016)
22. Chu, C. H. et al. Active dielectric metasurface based on phase-change medium Laser Photonics Rev. 10, 986-994 (2016).

23. Zhang, $\mathrm{H}$. et al. Topological insulators in Bi2Se3, Bi2Te3 and Sb2Te3 with a single Dirac cone on the surface. Nat. Phys. 5, 438-442 (2009).

24. Werner, W. S. M., Glantschnig, K. \& Ambrosch-Draxl, C. Optical constants and inelastic electron-scattering data for 17 elemental metals. J. Phys. Chem. Ref. Data 38, 1013-1092 (2009).

25. Yin, J. et al. Plasmonics of topological insulators at optical frequencies. NPG Asia Mater. 9, e425 (2017).

26. Barnes, W. L., Dereux, A. \& Ebbesen, T. W. Surface plasmon subwavelength optics. Nature 424, 824-830 (2003).

27. Dastmalchi, B., Tassin, P., Koschny, T. \& Soukoulis, C. M. A new perspective on plasmonics: confinement and propagation length of surface plasmons for different materials and geometries. Adv. Opt. Mater. 4, 177-184 (2015).

28. Naik, G. V., Shalaev, V. M. \& Boltasseva, A. Alternative plasmonic materials: beyond gold and silver. Adv. Mater. 25, 3264-3294 (2013).

29. Kats, M. A., Blanchard, R., Genevet, P. \& Capasso, F. Nanometre optical coatings based on strong interference effects in highly absorbing media. Nat. Mater. 12, 20-24 (2012).

30. Orava, J., Greer, A. L., Gholipour, B., Hewak, D. W. \& Smith, C. E. Characterization of supercooled liquid Ge2Sb2Te5 and its crystallization by ultrafast-heating calorimetry. Nat. Mater. 11, 279-283 (2012).

31. Liu, Y., Aziz, M. M., Shalini, A., Wright, C. D. \& Hicken, R. J. Crystallization of Ge2Sb2Te5 films by amplified femtosecond optical pulses. J. Appl. Phys. 112, 123526 (2012).

32. Wang, Q. et al. $1.7 \mathrm{Gbit} / \mathrm{in}^{2}$ gray-scale continuous-phase-change femtosecond image storage. Appl. Phys. Lett. 104, 121105 (2014).

33. Vos, J. J. Colorimetric and photometric properties of a $2^{\circ}$ fundamental observer. Color Res. Appl. 3, 125-128 (1978).

34. Hosseini, P., Wright, C. D. \& Bhaskaran, H. An optoelectronic framework enabled by low-dimensional phase-change films. Nature $\mathbf{5 1 1}$ 206-211 (2014)

35. Schlich, F. F., Zalden, P., Lindenberg, A. M. \& Spolenak, R. Color switching with enhanced optical contrast in ultrathin phase-change materials and semiconductors induced by femtosecond laser pulses. ACS Photonics 2, 178-182 (2015).

36. Dong, W., Qiu, Y., Yang, J., Simpson, R. E. \& Cao, T. Wideband absorbers in the visible with ultrathin plasmonic-phase change material nanogratings. J. Phys. Chem. C 120, 12713-12722 (2016).

37. Ríos, C., Hosseini, P., Taylor, R. A. \& Bhaskaran, H. Color depth modulation and resolution in phase-change material nanodisplays. Adv. Mater. 28, 4720-4726 (2016).

38. Hägglund, C., Apell, S. P. \& Kasemo, B. Maximized optical absorption in ultrathin films and its application to plasmon-based two-dimensional photovoltaics. Nano Lett. 10, 3135-3141 (2010).

39. Michel, A.-K. U., Wuttig, M. \& Taubner, T. Design parameters for phase-change materials for nanostructure resonance tuning. Adv. Opt. Mater. 5, 1700261 (2017).

40. Johnson, P. B. \& Christy, R. W. Optical constants of the noble metals. Phys. Rev. B $6,4370-4379$ (1972) 